\title{
In vivo reflectance confocal microscopy of wounds: feasibility of intraoperative basal cell carcinoma margin assessment
}

\author{
Veronika Shavlokhova $^{1}$, Michael Vollmer ${ }^{1}$, Andreas Vollmer ${ }^{1}$, Patrick Gholam ${ }^{2}$, Babak Saravi ${ }^{3}$, Jürgen \\ Hoffmann $^{1}$, Michael Engel ${ }^{1}$, Jens Elsner ${ }^{4}$, Florian Neumeier ${ }^{5}$, Christian Freudlsperger ${ }^{1}$ \\ ${ }^{1}$ Department of Oral and Maxillofacial Surgery, University Hospital Heidelberg, Heidelberg, Germany; ${ }^{2}$ Department of Dermatology, University \\ Hospital Heidelberg, Heidelberg, Germany; ${ }^{3}$ Department of Orthopedics and Trauma Surgery, Medical Centre-Albert-Ludwigs-University of \\ Freiburg, Faculty of Medicine, Albert-Ludwigs-University of Freiburg, Freiburg, Germany; ${ }^{4}$ Munich Innovation Labs GmbH, Munich, Germany; \\ ${ }^{5} \mathrm{M} 3 \mathrm{i}$ GmbH, Munich, Germany \\ Contributions: (I) Conception and design: V Shavlokhova, C Freudlsperger, J Hoffmann; (II) Administrative support: V Shavlokhova, C Freudlsperger, \\ J Hoffmann; (III) Provision of study materials or patients: M Vollmer, V Shavlokhova, P Gholam, M Engel, J Elsner, F Neumeier, C Freudlsperger; \\ (IV) Collection and assembly of data: V Shavlokhova, M Vollmer, C Freudlsperger; (V) Data analysis and interpretation: V Shavlokhova, M Vollmer, \\ A Vollmer, B Saravi; (VI) Manuscript writing: All authors; (VII) Final approval of manuscript: All authors. \\ Correspondence to: Veronika Shavlokhova. Department of Oral and Maxillofacial Surgery, University Hospital Heidelberg, Im Neuenheimer Feld 400, \\ 69120 Heidelberg, Germany. Email: veronika.shavlokhova@med.uni-heidelberg.de.
}

Background: In vivo reflectance confocal microscopy (RCM) is well established in non-melanoma skin cancer detection and screening. However, there is no sufficient validation regarding intraoperatively obtained images of wound margins. A reliable and fast resection margin detection is of high clinical relevance. Hence, we aimed to investigate feasibility and validity of in vivo RCM imaging for wound margins assessment compared with standard skin surface imaging and the gold standard histopathology.

Methods: A surgical incision through the center of a large basal cell carcinoma (BCC) affected area in the head and face region was performed. After removing half of the tumor, the wound margins of the remaining half as well as the corresponding skin surface were scanned with an in vivo RCM. A total of 50 wound margin images with BCC, 50 images of BCC-free margins and the corresponding skin surface images from 50 patients were compared with each other and with histopathological findings. Presence of confocal diagnostic criteria for BCC in images was analyzed.

Results: An overall sensitivity and specificity in detection of BCC in wound margins was $88.5 \%$, and $91.7 \%$ compared to skin surface imaging and $97.8 \%$ and $90.7 \%$, respectively, compared to histopathology. We identified all known confocal patterns of healthy skin and BCC in wound margin scans: damage of the epidermal layer above the lesion and cellular pleomorphism, elongated and monomorphic basaloid nuclei, nuclear polarization, an increased number of dilated blood vessels with high leukocyte traffic, inflammatory cells.

Conclusions: The accuracy of in vivo RCM imaging of wound margins is comparable with a standard skin surface imaging. The intraoperative detection of BCC areas in wound margins is as precise as the standard skin imaging and may be supportive for surgical interventions.

Keywords: Basal cell carcinoma (BCC); in vivo; reflectance confocal microscopy (RCM); margin assessment

Submitted Jul 02, 2021. Accepted for publication Sep 22, 2021.

doi: $10.21037 /$ atm-21-3462

View this article at: https://dx.doi.org/10.21037/atm-21-3462 


\section{Introduction}

The most common malignant tumour in the white population is basal cell carcinoma (BCC) and accounts for $75 \%$ of all non-melanoma skin cancers (1). Reports have shown that rates of BCC have increased in many countries around the world $(2,3)$. This can be explained by increasing life expectancy and exposure to sunlight. A precise description of the epidemiology of BCC is difficult. Since most cancer registries only register the first histologically confirmed BCC per patient, the true incidence of BCC is probably significantly underestimated (4). BCC is most common in adults, particularly in the elderly population, although it has recently been reported frequently in adults under 50 years of age (5). Risk factors for BCC disease are, in addition to light pigment characteristics (light skin, red hair), an older age, genodermatoses, a family history of the disease and immunosuppression (6). Furthermore, organ transplant patients represent a special risk group. Population-based studies have shown that patients who have undergone immunosuppression due to transplantation have up to a 16-fold increased risk of developing BCC. Kidney transplant patients showed the highest risk $(7,8)$.

Mohs surgery is considered as an effective and tissuesparing therapeutical approach for nonmelanoma skin cancers (1). The technique gained increasingly popular especially due to its minimal-invasiveness, considering that these types of tumors often occur in functional and aesthetic relevant regions exposed to the sun (e.g., head and neck area). Tissue specimens are removed during surgery and rapidly frozen and sectioned using a cryostat microtome for fast processing. Although this technique allows guidance of the surgery through examination of frozen sections during surgery until margins show no residuals of tumor histologically, the procedure requires tissue preparation which can take 20-45 minutes per excision (2).

In vivo reflectance confocal microscopy (RCM) is a noninvasive real-time high-resolution technique for optical imaging with a reported sensitivity up to $100 \%$ and specificity up to $88 \%$ for the diagnosis of BCC (9-11). Considering available diagnostic data, RCM might be a promising future method for detection and screening of tumors. It has been already reported for preoperative use (9). However, reliable results were only achieved with superficial and nodular types. Preoperative application is not reliable for tumours located deep below the superficial dermis (12). Notably, there are not many studies on the applicability of intraoperative confocal microscopy to assess the wound margins $(9,13)$ and there are no studies comparing this imaging technique with standard skin surface imaging. Kang et al. recently presented a multichannel confocal fluorescence microscopy and showed that the focus tracking and line scanning technique allow a large field of and depth of detail compared to H\&E histology (14). A high-speed form of RCM, the spectrally encoded confocal microscopy (SECM), was introduced by the workgroup of Yoo et al. and even allowed to count intraepithelial cells reliably without administration of exogenous contrasts (15).

Furthermore, feasibility of direct imaging of BCC tumour residues at the surgical margins has already been demonstrated previously (9). However, this required topical application of aluminium chloride ( $\mathrm{AlCl}$ ) to optimise nuclear morphology brightening and BCC tumour-to-skin contrast (9). Misrepresented nuclear morphology and tissue necrosis could result depending on the concentration used $(9,16)$. Moreover, they harbour risks for side effects $(16)$.

The standard skin surface imaging protocol includes following steps. First, the skin is being mechanically fixed to minimize its motion, although small movements are also being tolerated. After being vertically displaced on intact skin surface, the lens changes the depth of the image within the tissue, and the system software facilitates the collection of sequential XY planes of the scanned area.

Compared to standard in vivo RCM skin surface imaging protocols, there are some additional challenges for wound margin scanning. First, the wound shape and surface of its base are irregular: imaging is to be performed along three layers: dermal, epidermal and the wound base. Another problem is bleeding which can result in scanning artifacts. Further, hemostasis through topical application of vasoconstrictors or electrocautery can lead to misinterpretation of confocal images.

Known in vivo RCM features of BCC include the presence of compact aggregates of a uniform population of tumor cells (17-19). Their nuclei are elongated and oriented along the same axis, a feature termed "nuclear polarization" $(18,20,21)$. Nodular BCC has refractile islands with a line of basaloid nuclei organized perpendicular to the axis of the tumor aggregate, forming a "peripheral nuclear palisade" $(18,22)$. These aggregates are sometimes surrounded by dark clefts that separate them from the surrounding stromal tissue (23). Around the tumor, the stroma shows highly refractile collagen bundles with frayed edges. Likewise, vascularization is increased and blood vessels are dilated $(19,24)$.

Here, we aimed to evaluate the feasibility of in vivo RCM on BCC wound margin diagnostics compared with standard 
skin surface imaging protocols and histopathology. We present the following article in accordance with the STARD reporting checklist (available at https://dx.doi.org/10.21037/ atm-21-3462).

\section{Methods}

\section{Patients}

Confocal microscopic images of BCCs were taken at the Department of Oral and Maxillofacial Surgery (Heidelberg University Hospital). The study was conducted according to the guidelines of the Declaration of Helsinki (as revised in 2013) and approved by the Institutional Ethics Committee of Heidelberg University (protocol code S-665/2019). Participants gave informed consent before taking part. All data were obtained prospectively and saved in a pseudonymised form on a hard drive; information on patients' data (age, sex, histopathological report) was saved in a separate database. The inclusion criteria were: (I) patients aged 18 years and older who gave a written informed consent; (II) participants with histologically proven BCC in the head and face region; (III) a resection of the lesion was indicated. The exclusion criteria were: (I) lesions smaller than $1 \mathrm{~cm}$ in diameter; (II) previous surgeries. We identified and selected a total of 50 patients with large histologically proven BCCs who gave the written consent to participate in the study. Eligible patients were included consecutively.

\section{Imaging}

From each patient, image sequences from large histologically proven BCC areas in the head and face region were taken with an in vivo RCM Vivascope 3000 (Mavig GmbH, Munich, Germany).

This confocal microscope is a light microscope that consists of a light source, condenser, lens, diaphragm and detector. The point of origin of the light source is located in a focal plane conjugated to that of the tissue plane under examination. Images correspond to transversal optical sections of the tissue, which are parallel to the surface of the skin. These images emerge from the reflected light collected only from the tissue plane under study (confocal plane), as it is scanned by the laser light source. Before reaching the detector, the light reflected by the tissue goes through a diaphragm located in a plane conjugated to that of the light source and the focal plane. An inversely proportional relationship exists between axial resolution and pinhole aperture $(25,26)$. RCM uses a laser as a light source and the wavelength for maximum lateral resolution and optimization of the reflectance capability. An optimized setup includes a low power laser $(30 \mathrm{~mW})$ that emits a coherent beam of near-infrared wavelength (800-1,064 nm) (27). The RCM achieves a resolution of $1.25 \mu \mathrm{m}$ with a vertical resolution of $5 \mu \mathrm{m}$ in the target field of view (FOV). A maximum penetration depth of approx. $350 \mu \mathrm{m}$ is achievable $(28,29)$. This is aimed to take advantage of the higher reflectance of melanin-containing structures, as its refractive index (1.7) is higher than that of surrounding tissue (1.34), resulting in very good contrast (30). The optics of choice are water- or ultrasound gelimmersion lenses which prevent losses of contrast. Image contrast is provided by differences in the amount of light reflected by the tissue due to intrinsic differences in the refractive indices of the microscopic structures that are part of the skin. This eliminates the need for exogenous contrast.

The confocal images were taken according to the following protocol. First, surgical incision through the middle line of a lesion was performed. After removal of half of the tumour, the wound cavity was covered with a sterile transparent dressing (Tegaderm, 3M, St. Paul, MN, USA). To perform imaging, a drop of Crodamol STS oil (Croda Inc., Edison, NJ, USA) was applied to the dressing. Most of the wounds used to be anemic due to local anesthesia. If that was not the case, we coagulated all bleeding vessels electrically. Next, wound margins of the not resected tumour half as well as the corresponding skin surface areas were scanned (Figure 1).

During scanning, image stacks were generated, displaying $1 \mathrm{~mm} \times 1 \mathrm{~mm}$ FOV. Due to time restrictions, the full wound and margin surface was not imaged. We selected the peripheral epidermal areas as the main representative imaging areas where we could provide the corresponding perpendicular skin surface imaging (Figure 2). The deep dermal margins and upper periphery were not imaged.

The confocal scans acquired during surgery, were blindly analyzed by two maxillofacial surgeons of the Oral and Maxillofacial Surgery Department of the University of Heidelberg in Germany, extensively trained in the field of confocal laser microscopy (VivaScope Expert Training Course, Prof. Dr. Giovanni Pellacani University of Modena and Reggio Emilia, Italy, Prof. Dr. Caterina Longo University of Modena and Reggio Emilia, Italy, Prof. Dr. Marco Ardigò San Gallicano Hospital, Italy).

The result of the investigation amounted a total of 50 wound margin images with BCC, 50 wound margin images 

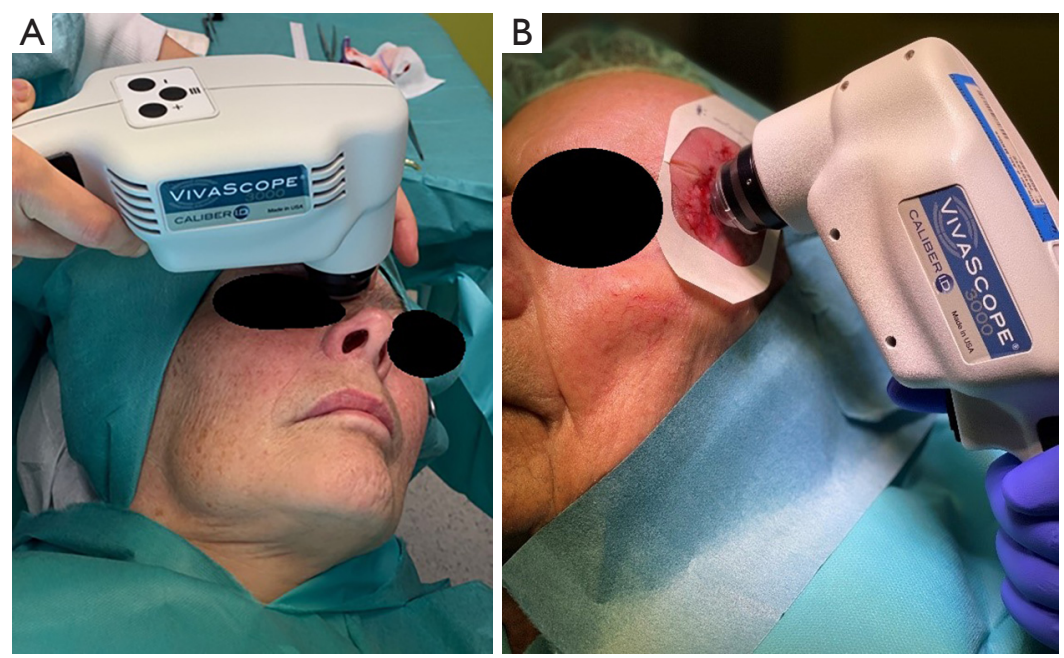

Figure 1 Intraoperative application and wound assessment with in vivo RCM scanning. This image is published with the patient's consent. RCM, reflectance confocal microscopy.

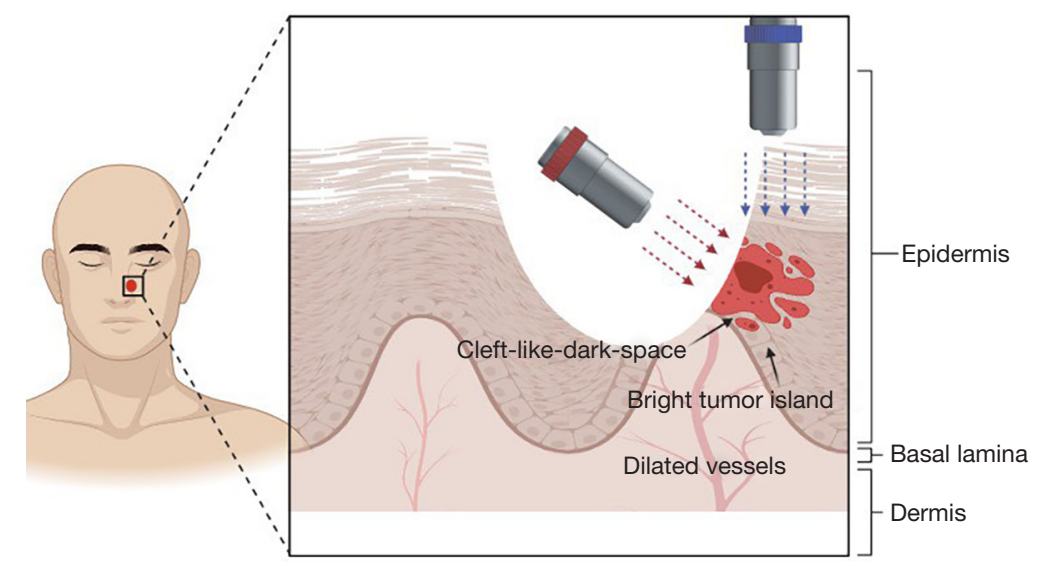

Figure 2 Illustration of surgical wound topography. Imaging was performed in two areas: (red lens) epidermal-dermal margin and (blue lens) corresponding skin surface area.

without BCC and 100 corresponding skin surface images from 50 patients which were selected for the evaluation.

Following established in vivo RCM diagnostic parameters of BCC ("confocal criteria") were evaluated in each confocal image:

* Damage of the epidermal layer above the lesion and cellular pleomorphism;

* Tumor cells with elongated and monomorphic basaloid nuclei;

* Alignment of all nuclei along the same axis ("nuclear polarization");

* An increased number of dilated blood vessels with high leukocyte traffic;
* Tumor associated inflammatory cells.

Histopathology was applied as the gold standard on all excisions. The scanned areas of resected tumors were marked with sutures to compare the report diagnosis with the results of scans.

The statistical analyses in the current study were performed using the SPSS 25.0 (IBM, Armonk, New York) and $\mathrm{R}$ version 3.6.2 (31,32). To evaluate the diagnostic accuracy of in vivo RCM in detecting BCC in wound margins, the sensitivity, specificity, positive predictive value (PPV) and negative predictive value (NPV) were calculated. True positive was defined as the presence of confocal microscopic criteria for BCC in both imaging modalities; 
true negative as no such findings in both; false negative as the presence of morphologic criteria for BCC in confocal imaging of skin surface, but no such findings in the wound margins; and false positive as the presence of these findings in the wound margin scans but with none in confocal imaging of skin.

\section{Results}

\section{Population}

Fifty patients with BCC of the head and face region were included in the study. Among them 27 were females and

Table 1 Patient characteristics

\begin{tabular}{lc}
\hline Parameter & Data \\
\hline Age, mean years [standard deviation] & $69[12]$ \\
Gender, $\mathrm{n}(\%)$ & \\
Female & $27(54 \%)$ \\
Male & $23(46 \%)$ \\
Location, $\mathrm{n}(\%)$ & \\
Forehead & $12(24 \%)$ \\
Head & $31(62 \%)$ \\
Cheek & $2(4 \%)$ \\
Nose & $4(8 \%)$ \\
Ear & $1(2 \%)$ \\
\hline
\end{tabular}

23 males. Mean age was $69 \pm 12$ years (Table 1 ). The majority of BCC in our study were nodular $(\mathrm{N}=33)$, followed by infiltrative $(\mathrm{N}=9)$ and superficial $(\mathrm{N}=8)$.

\section{BCCs detection accuracy}

The detection accuracy of BCCs in wound margin images compared to those taken from the skin surface showed a sensitivity (S) of $88.5 \%$, a specificity (Sp) of $91.7 \%$, a PPV of $92 \%$ and a NPV of $88 \%$ (Table 2). The same data compared to conventional H\&E staining (Table 3) showed the following values: S 97.8\%, Sp 90.7\%, PPV 90\%, NPV 98\%.

With the help of in vivo RCM we were able to correctly identify following confocal criteria of BCC in both the wound margins and corresponding skin surfaces: damage of the epidermal layer above the lesion and cellular pleomorphism, tumor cells with elongated and monomorphic basaloid nuclei, alignment of all nuclei along the same axis ("nuclear polarization"), an increased number of dilated blood vessels with high leukocyte traffic and tumor associated inflammatory cells (Table 4).

In terms of image quality, we observed good resolution, contrast and recognisability of the typical BCC criteria. Morphological guidance structures such as glands, hair follicles and inflammatory cells could be well visualised at the nuclear and cellular level (Figure 3).

\section{Evaluation of surgical wounds}

In both wound margin images with BCC and wound margin

Table 2 Detection of BCC in in vivo RCM wound margin images compared to those of skin surface

\begin{tabular}{lccc}
\hline In vivo FCM image characteristic & In vivo skin surface + & In vivo skin surface - & $\mathrm{N}(\%)$ \\
\hline In vivo RCM wound margins + & 46 & 6 & $52(52 \%)$ \\
In vivo RCM wound margins - & 4 & 44 & $48(48 \%)$ \\
$\mathrm{N}(\%)$ & $50(50 \%)$ & $50(50 \%)$ & $200(100 \%)$ \\
\hline
\end{tabular}

BCC, basal cell carcinoma; RCM, reflectance confocal microscopy.

Table 3 Detection of BCC in in vivo RCM wound margin images compared to those of histopathology

\begin{tabular}{lccc}
\hline In vivo FCM and H\&E characteristic & H\&E + & H\&E - & N (\%) \\
\hline In vivo RCM wound margins + & 45 & 1 & $46(46 \%)$ \\
In vivo RCM wound margins - & 5 & 49 & $54(54 \%)$ \\
$\mathrm{N}(\%)$ & $50(50 \%)$ & $50(50 \%)$ & $100(100 \%)$ \\
\hline
\end{tabular}

BCC, basal cell carcinoma; RCM, reflectance confocal microscopy. 
Table 4 Evaluation of presence of BCC in vivo RCM criteria in wound margins

\begin{tabular}{|c|c|c|}
\hline $\mathrm{BCC}$ In vivo $\mathrm{RCM}$ criteria & $\begin{array}{c}\text { Present }[\%] \text { in BCC wound } \\
\text { margins scans }\end{array}$ & $\begin{array}{c}\text { Present [\%] in BCC skin } \\
\text { surface scans }\end{array}$ \\
\hline Damage of the epidermal layer above the lesion and cellular pleomorphism & $47[94]$ & $50[100]$ \\
\hline Alignment of all nuclei along the same axis ("nuclear polarization") & $50[100]$ & $50[100]$ \\
\hline An increased number of dilated blood vessels with high leukocyte traffic & $27[54]$ & $43[86]$ \\
\hline
\end{tabular}

BCC, basal cell carcinoma; RCM, reflectance confocal microscopy.

images without BCC a good imaging quality comparable with that of standard intact skin imaging was observed for all examined samples.

Following wound levels could be investigated with a confocal microscope and described: epidermal margin, papillary dermis layer and the wound base. In epidermal margins, typical cobblestone patterns representing epidermal keratinocytes could be found. These structures are typically displayed as a honeycomb pattern when scanned perpendicularly from the skin surface. In the deeper papillary layer, the dermoepidermal junction with papillae. These papillae appear as dark areas containing blood vessels and refractile collagen fibers. The basal keratinocytes appear as vertically orientated cascades, in contrast to the ring form if scanned from intact skin. In the base of a wound, papillary dermis and reticular dermis structures could be observed. These layers appeared darker than the dermis with no visible nuclei and refractile collagen fibres.

\section{Discussion}

After previous initial work on intraoperative in vivo RCM imaging, we validated the method for application on wound margins by comparing it with standard intact skin scanning and the gold standard histopathology. This study included a substantial of surgical tumour resection wounds $(\mathrm{N}=50)$. The device used in our work is the newest version of Vivascope confocal microscopes with a relatively small handheld scanning part, providing the best possible speed of investigation of large areas. Compared to previous studies on the topic, we did not use any contrast agent to increase the visibility of nuclear morphology, as the main confocal criteria of BCC were easy to recognize "natively" (9). We were able to avoid additional contrast agents such as $\mathrm{AlCl}$, indocyanine green (ICG), sodium fluorescein and acetic acid and thus avoid further potential sources of error and side effects as well as a greater time delay for the measurement $(9,16)$.

Contrast enhancing agents are associated with several disadvantages, including increased time consumption, error-proneness, and discolouration of the skin with artefact formation (16). Omission of this intermediate step allows for a faster and clinically easier to establish approach with fewer sources of error.

The present study did not assess the coverage rate, as we preferably selected larger lesions and scanning the whole surface would have quite prolonged the operating time. Furthermore, the irregularity of each wound surface could lead to incorrect estimation.

Determining epidermal margin, papillary dermis layer and the wound base was possible in all cases, as all characteristic layer structures could be well-identified. Similarly, scanning of BCC containing wound margins allowed to determine the aforementioned structures.

The present version of Vivascope [3000] confocal microscope represents the most maneuverable handheld version of all generations, which enables scanning of difficult to access regions, like the nose or the ears (29). In our study, the device could also scan the surface of crater shaped wounds. To provide a precise comparison of margin areas and the corresponding imaging of skin surface, we included rather large tumors of at least $2-3 \mathrm{~cm}$ diameter. However, a smaller objective lens of confocal scanner may also ensure a precise imaging in the smallest wounds or defined areas of interest in margins. An automated approach is another desirable feature for an objective, quick and complete scanning.

Levine and Markowitz [2018] already described the attempt of an automated process to identify predefined mosaics from several tissue depths (33). Sufficiently identifying and evaluating the individual layers requires 


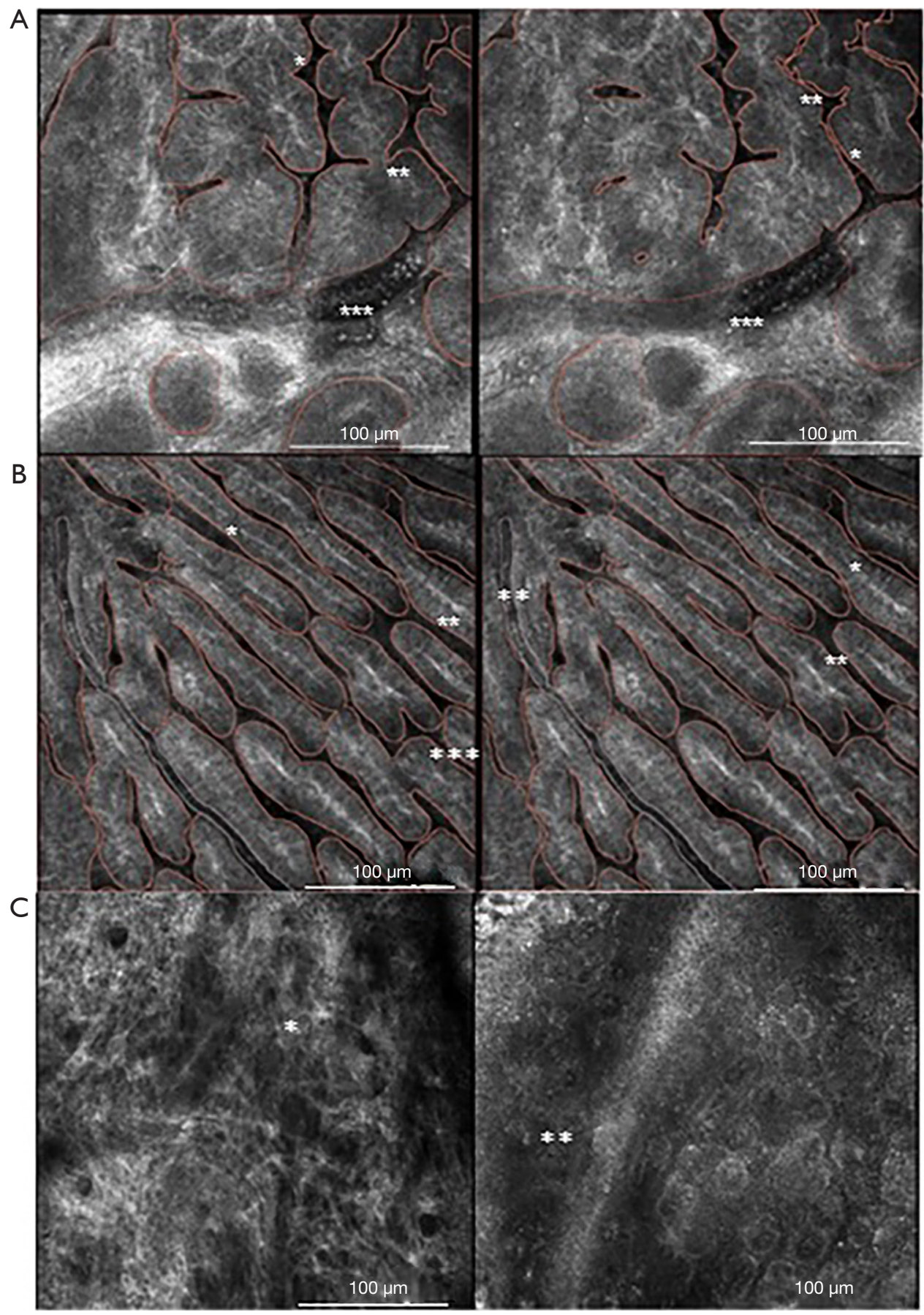

Figure 3 In vivo RCM images. Confocal microscope image BCC frontal (first image $22.8 \mu \mathrm{m}$, second image $27.36 \mu \mathrm{m}$ ) (blue lens in Figure 2) scanned according to the standard protocol from the skin surface (A), confocal microscope image BCC lateral (first image $45.6 \mu \mathrm{m}$, second image $50.16 \mu \mathrm{m}$ ) (red lens in Figure 2), the view with a confocal microscope where the lens is placed directly on the wound on the epidermaldermal margin (B) and healthy tissue (first image $4.56 \mu \mathrm{m}$ second image $13.68 \mu \mathrm{m}$ ), a lateral scan (C). *, tumor cells with elongated and monomorphic basaloid nuclei; **, alignment of all nuclei along the same axis ("nuclear polarization"); ***, tumor associated inflammatory cells. RCM, reflectance confocal microscopy; BCC, basal cell carcinoma. 
extensive training of the examiner. Overall, they concluded that the risk of missing clinically important levels increases with growing tissue depth (33).

Kurogol et al. [2015] have shown that automated algorithms can support an objective interpretation of structures with regards to dermal-epidermal-junction identification utilizing RCM (34).

Training an artificial intelligence could make a significant advance in this area in terms of objectification and reproducibility.

In our study we used the lens-to-tissue contact ring for skin surface imaging and covered the wound with Tagaderm film to provide a sterile environment. The expected artifacts caused by covering the wound with a sterile Tegaderm as a barrier to the objective lens, were rather minor and had no or a small impact on image quality. Those artifacts were: wrinkling and folding of Tagaderm film and presence of air bubbles. Nevertheless, a contact ring allowing lensto-wound confocal imaging might be profitable in terms of avoiding additional artifacts and reducing the whole imaging time.

\section{BCCs detection accuracy}

Using in vivo RCM, we were able to correctly identify the presence of BCC in wound margins with a sensitivity of $88.5 \%$ and a specificity of $91.7 \%$, compared to in vivo RCM of intact skin. These results are in agreement with previously published pilot studies on feasibility of wound scanning $(9,13,29,35-37)$. We hypothesized that in vivo RCM of intact skin and wound margins might be very similar, but expected a number of imaging artifacts, like those caused by intraoperative bleeding. However, the bleeding was rather absent due to application of local anaesthesia. All actively bleeding vessels in our study were coagulated and in none of our cases image interpretation was affected due to bleeding. Interestingly, dilated vessels as one of the in vivo confocal criteria of BCC reported by other authors could only be found in $27(54 \%)$ cases $(19,24)$. This might be due to the application of local anesthetics containing a vasoconstrictor. In contrast, dilated vessels already described for presurical imaging by other authors could be observed in 43 (86\%) of corresponding skin images $(29,30)$.

In all cases of both imaging modalities (wound margins and intact skin), tumor cells with elongated and monomorphic basaloid nuclei and alignment of all nuclei along the same axis could be easily found. From previous works we know that combination of these two criteria (identification of nuclear polarisation and elongated nuclei) is the most sensitive (91.6\%) and specific (97.1\%) for in vivo RCM diagnosis of BCC $(18,19,24,29,38)$.

In our work we did not aim to characterize the subtype of BCC. In our opinion, the intraoperative wound imaging is primarily a possible support to determine the optimal resection, based on a quick detection of residual BBC areas. Nevertheless, the differences of BCC subtypes were described in previous works $(18,19,39)$. Furthermore, Kadouch et al. [2017] have already shown that, depending on the experience of RCM examiner, there is a comparable diagnostic accuracy in the subclassification of BCC entities (40). Limitations of the present preliminary work mainly include the small sample size ( $\mathrm{n}=50$ per group) and the inclusion of only large tumors $(\geq 2-3 \mathrm{~cm})$ in our study. A multicenter study utilizing a large number of different types of BCC, and the use of small lenses for RCM is warranted in future to allow a more precise estimation of the diagnostic value for application in clinics.

In conclusion, the present study continues a series of initial works on in vivo RCM wound imaging and emphasizes the expandability of this technology which was primarily developed for intact skin imaging. The sensitivity and specificity for BCC detection in wound margins is as high as that for skin surface in vivo RCM imaging. This was assessed on a collective of 50 patients and validated through comparison with corresponding in vivo skin images and histopathology as gold standard. The reported approach serves a good basis for further developments like automated classification and AI based detection of cancerous tissue.

\section{Acknowledgments}

The Vivascope device was provided by Mavig GmbH for the time of the study.

Funding: This research was funded by Federal Ministry of Education and Research, grant number 13GW0362D.

\section{Footnote}

Reporting Checklist: The authors have completed the STARD reporting checklist. Available at https://dx.doi. org/10.21037/atm-21-3462

Data Sharing Statement: Available at https://dx.doi. org/10.21037/atm-21-3462

Conflicts of Interest: All authors have completed the ICMJE uniform disclosure form (available at https://dx.doi. 
org/10.21037/atm-21-3462). The authors have no conflicts of interest to declare.

Ethical Statement: The authors are accountable for all aspects of the work in ensuring that questions related to the accuracy or integrity of any part of the work are appropriately investigated and resolved. The study was conducted according to the guidelines of the Declaration of Helsinki (as revised in 2013) and approved by the Institutional Ethics Committee of Heidelberg University (protocol code S-665/2019). Participants gave informed consent before taking part.

Open Access Statement: This is an Open Access article distributed in accordance with the Creative Commons Attribution-NonCommercial-NoDerivs 4.0 International License (CC BY-NC-ND 4.0), which permits the noncommercial replication and distribution of the article with the strict proviso that no changes or edits are made and the original work is properly cited (including links to both the formal publication through the relevant DOI and the license). See: https://creativecommons.org/licenses/by-nc-nd/4.0/.

\section{References}

1. Dessinioti C, Antoniou C, Katsambas A, et al. Basal cell carcinoma: what's new under the sun. Photochem Photobiol 2010;86:481-91.

2. Cameron MC, Lee E, Hibler BP, et al. Basal cell carcinoma: Epidemiology; pathophysiology; clinical and histological subtypes; and disease associations. J Am Acad Dermatol 2019;80:303-17.

3. Joseph AK, Mark TL, Mueller C. The period prevalence and costs of treating nonmelanoma skin cancers in patients over 65 years of age covered by medicare. Dermatol Surg 2001;27:955-9.

4. Richmond-Sinclair NM, Pandeya N, Ware RS, et al. Incidence of basal cell carcinoma multiplicity and detailed anatomic distribution: longitudinal study of an Australian population. J Invest Dermatol 2009;129:323-8.

5. Asgari MM, Moffet HH, Ray GT, et al. Trends in Basal Cell Carcinoma Incidence and Identification of High-Risk Subgroups, 1998-2012. JAMA Dermatol 2015;151:976-81.

6. Hogan DJ, To T, Gran L, et al. Risk factors for basal cell carcinoma. Int J Dermatol 1989;28:591-4.

7. Moloney FJ, Comber H, O'Lorcain P, et al. A populationbased study of skin cancer incidence and prevalence in renal transplant recipients. Br J Dermatol 2006;154:498-504.
8. Krynitz B, Olsson H, Lundh Rozell B, et al. Risk of basal cell carcinoma in Swedish organ transplant recipients: a population-based study. Br J Dermatol 2016;174:95-103.

9. Flores ES, Cordova M, Kose K, et al. Intraoperative imaging during Mohs surgery with reflectance confocal microscopy: initial clinical experience. J Biomed Opt 2015;20:61103.

10. Webber SA, Wurm EM, Douglas NC, et al. Effectiveness and limitations of reflectance confocal microscopy in detecting persistence of basal cell carcinomas: a preliminary study. Australas J Dermatol 2011;52:179-85.

11. Marra DE, Torres A, Schanbacher CF, et al. Detection of residual basal cell carcinoma by in vivo confocal microscopy. Dermatol Surg 2005;31:538-41.

12. Pan ZY, Lin JR, Cheng TT, et al. In vivo reflectance confocal microscopy of Basal cell carcinoma: feasibility of preoperative mapping of cancer margins. Dermatol Surg 2012;38:1945-50.

13. Scope A, Mahmood U, Gareau DS, et al. In vivo reflectance confocal microscopy of shave biopsy wounds: feasibility of intraoperative mapping of cancer margins. $\mathrm{Br}$ J Dermatol 2010;163:1218-28.

14. Kang B, Afifi MM, Austin LA, et al. Exploiting the nanoparticle plasmon effect: observing drug delivery dynamics in single cells via Raman/fluorescence imaging spectroscopy. ACS Nano 2013;7:7420-7.

15. Yoo H, Kang D, Katz AJ, et al. Reflectance confocal microscopy for the diagnosis of eosinophilic esophagitis: a pilot study conducted on biopsy specimens. Gastrointest Endosc 2011;74:992-1000.

16. Ring HC, Israelsen NM, Bang O, et al. Potential of contrast agents to enhance in vivo confocal microscopy and optical coherence tomography in dermatology: A review. J Biophotonics 2019;12:e201800462.

17. Sahu A, Yélamos O, Iftimia N, et al. Evaluation of a Combined Reflectance Confocal Microscopy-Optical Coherence Tomography Device for Detection and Depth Assessment of Basal Cell Carcinoma. JAMA Dermatol 2018;154:1175-83.

18. Peppelman M, Wolberink EA, Blokx WA, et al. In vivo diagnosis of basal cell carcinoma subtype by reflectance confocal microscopy. Dermatology 2013;227:255-62.

19. Lupu M, Popa IM, Voiculescu VM, et al. A Retrospective Study of the Diagnostic Accuracy of In Vivo Reflectance Confocal Microscopy for Basal Cell Carcinoma Diagnosis and Subtyping. J Clin Med 2019;8:449.

20. González S. Confocal reflectance microscopy in dermatology: promise and reality of non-invasive diagnosis and monitoring. 


\section{Page 10 of 10}

Actas Dermosifiliogr 2009;100 Suppl 2:59-69.

21. Calzavara-Pinton P, Longo C, Venturini M, et al. Reflectance confocal microscopy for in vivo skin imaging. Photochem Photobiol 2008;84:1421-30.

22. González S, Tannous Z. Real-time, in vivo confocal reflectance microscopy of basal cell carcinoma. J Am Acad Dermatol 2002;47:869-74.

23. Ulrich M, Roewert-Huber J, González S, et al. Peritumoral clefting in basal cell carcinoma: correlation of in vivo reflectance confocal microscopy and routine histology. J Cutan Pathol 2011;38:190-5.

24. Nori S, Rius-Díaz F, Cuevas J, et al. Sensitivity and specificity of reflectance-mode confocal microscopy for in vivo diagnosis of basal cell carcinoma: a multicenter study. J Am Acad Dermatol 2004;51:923-30.

25. Cameron MC, Rossi AM. Reflectance Confocal Microscopy for Skin Cancer Margins: How it Works. Curr Derm Rep. 1. September 2016;5(3):172-8.

26. Nwaneshiudu A, Kuschal C, Sakamoto FH, et al. Introduction to confocal microscopy. J Invest Dermatol 2012;132:e3.

27. Que SK, Fraga-Braghiroli N, Grant-Kels JM, et al. Through the looking glass: Basics and principles of reflectance confocal microscopy. J Am Acad Dermatol 2015;73:276-84.

28. Fraga-Braghiroli NA, Stephens A, Grossman D, et al. Use of handheld reflectance confocal microscopy for in vivo diagnosis of solitary facial papules: a case series. J Eur Acad Dermatol Venereol 2014;28:933-42.

29. Edwards SJ, Osei-Assibey G, Patalay R, et al. Diagnostic accuracy of reflectance confocal microscopy using VivaScope for detecting and monitoring skin lesions: a systematic review. Clin Exp Dermatol 2017;42:266-75.

30. Venturini $M$, Gualdi G, Zanca A, et al. A new approach for presurgical margin assessment by reflectance confocal microscopy of basal cell carcinoma. Br J Dermatol 2016;174:380-5.

31. IBM SPSS Statistics 25 [Internet]. [zitiert 11. Mai 2021]. Available online: https://www.ibm.com/support/pages/ downloading-ibm-spss-statistics-25

32. R-3.6.2 for Windows [Internet]. 2019 [zitiert 11. Mai 2021]. Available online: https://cran.r-project.org/bin/ windows/base/old/3.6.2/

33. Levine A, Markowitz O. Introduction to reflectance confocal microscopy and its use in clinical practice. JAAD Case Rep 2018;4:1014-23.

34. Kurugol S, Kose K, Park B, et al. Automated delineation of dermal-epidermal junction in reflectance confocal

\section{Shavlokhova et al. Detection of tumour residuals in wound margins}

microscopy image stacks of human skin. J Invest Dermatol 2015;135:710-7.

35. Flores ES, Cordova M, Kose K, et al. Feasibility of intraoperative imaging during Mohs surgery with reflectance confocal microscopy. In: Photonic Therapeutics and Diagnostics X [Internet]. International Society for Optics and Photonics; 2014 [zitiert 13. Mai 2021]. S. 89260F. Available online: https://www.spiedigitallibrary.org/ conference-proceedings-of-spie/8926/89260F/Feasibilityof-intraoperative-imaging-during-Mohs-surgery-withreflectance-confocal/10.1117/12.2039590.short

36. Flores E, Yélamos O, Cordova M, et al. Peri-operative delineation of non-melanoma skin cancer margins in vivo with handheld reflectance confocal microscopy and video-mosaicking. J Eur Acad Dermatol Venereol 2019;33:1084-91.

37. Flores E, Yelamos O, Cordova M, et al. Peri-operative imaging of cancer margins with reflectance confocal microscopy during Mohs micrographic surgery: feasibility of a video-mosaicing algorithm. In: Photonics in Dermatology and Plastic Surgery [Internet]. International Society for Optics and Photonics; 2017 [zitiert 11. Mai 2021]. S. 100370B. Available online: https://www.spiedigitallibrary.org/conferenceproceedings-of-spie/10037/100370B/Peri-operativeimaging-of-cancer-margins-with-reflectance-confocalmicroscopy/10.1117/12.2252440.short

38. Castro RP, Stephens A, Fraga-Braghiroli NA, et al. Accuracy of in vivo confocal microscopy for diagnosis of basal cell carcinoma: a comparative study between handheld and wide-probe confocal imaging. J Eur Acad Dermatol Venereol 2015;29:1164-9.

39. Longo C, Lallas A, Kyrgidis A, et al. Classifying distinct basal cell carcinoma subtype by means of dermatoscopy and reflectance confocal microscopy. J Am Acad Dermatol 2014;71:716-724.e1.

40. Kadouch DJ, Leeflang MM, Elshot YS, et al. Diagnostic accuracy of confocal microscopy imaging vs. punch biopsy for diagnosing and subtyping basal cell carcinoma. J Eur Acad Dermatol Venereol 2017;31:1641-8.

Cite this article as: Shavlokhova V, Vollmer M, Vollmer A, Gholam P, Saravi B, Hoffmann J, Engel M, Elsner J, Neumeier F, Freudlsperger C. In vivo reflectance confocal microscopy of wounds: feasibility of intraoperative basal cell carcinoma margin assessment. Ann Transl Med 2021;9(23):1716. doi: 10.21037/ atm-21-3462 